Research Article; Received: March 03, 2020; Accepted: July 8, 2021

\title{
THE EFFECT OF SEMI PERFORATED DUCT ON RING SOURCED ACOUSTIC DIFFRACTION
}

\author{
Burhan TIRYAKIOGLU \\ Department of Applied Mathematics, Marmara University, Istanbul, TURKEY
}

\begin{abstract}
An analytical solution is obtained for the diffraction problem. In an infinite cylindrical duct, the sound waves are emanating by a ring source. The duct is rigid for $z<l$ and perforated for $z>l$. The mixed boundary value problem is defined by a Wiener Hopf equation, by using the Fourier transform technique. Then the numerical solution is obtained. The influence of the parameters of the problem on the diffraction phenomenon is displayed graphically. The present study can be used as a model for different applications. Reducing noise in exhaust systems, ventilation systems are some of these applications.
\end{abstract}

\section{INTRODUCTION}

Radiation or diffraction of sound waves is an essential problem which has been extensively studied in the literature so far. The duct and pipe structures are commonly used in many industrial devices to control the unwanted and harmful noise, such as exhaust systems, ventilation systems, aircraft jet and modern turbofan engines. For this reason, it is essential to investigate more accurate mathematical models for such engineering problems.

The radiation of sound waves from a semi-infinite rigid duct was first discussed by [1]. In their study, by using the Wiener-Hopf technique, the solution was obtained analytically [2. Covering the pipe/duct walls with an absorbing lining is an efficient method that has proven beneficial in noise reduction [3]. Another method of reducing unwanted sound is to provide additional sound absorption by using perforated structures. The phenomenon of perforated structures has been investigated by various authors $[6[10]$. This idea is essential because perforated structures provide some possibilities for investigating of sound diffraction.

2020 Mathematics Subject Classification. Primary 47A68, Secondary 42B10, 78A45.

Keywords. Wiener-Hopf, ring source, perforated, duct, saddle point.

• burhan.tiryakioglu@marmara.edu.tr

(D) 0000-0003-1448-6147. 
The goal of the present study is to consider the diffraction of sound waves emanating from a ring source by an infinite circular cylindrical duct. Duct walls are assumed to be infinitely thin and rigid for $z<l$, perforated for $z>l$. The ring source is located out of the duct and can be moved along the duct axis, but never go beyond the duct exit. This problem is a generalization of a previous work by the author [11 who considered the similar geometry in the case where the numerical results is absent. This geometry can be considered as a model of an acoustic waveguide for noise reduction. Due to the ring source, the total field have angular symmetry which makes the problem simpler than the asymmetric case. This mixed boundary value problem is investigated rigorously through the Wiener-Hopf technique. By applying the Fourier transform, we obtain a Wiener-Hopf equation. After application of usual decomposition and factorisation procedures the solution of the Wiener-Hopf equation is obtained. Then, numerical solution is obtained approximately for various values of the parameters such as the radii of the duct and ring source, frequency, perforated part, etc. The effect of values of the parameters on the diffraction phenomenon is presented graphically.

Validation of graphical results is obtained with unperforated (open) case. When the perforated part is absent, the present study is compared with the study of 12 and the results are found to be in good agreement.

\section{ANALYSIS}

2.1. Formulation of the Problem. We consider the diffraction of sound waves by an infinite cylindrical duct. Infinitely thin duct walls are assumed. The duct is $\{r=a, z \in \mathbb{R}\}$ illuminated by a ring source located at $\{r=b>a, z=-c, c>0\}$ (see Fig. 1). The part $z<l$ of the inner cylinder is hard walled while the part $z>l$ is perforated. From the installation of the problem, the ring source and the geometry is symmetrical. Therefore, the total field will be independent of azimuth $\phi$ everywhere in coordinate system $(r, \phi, z)$. The velocity potential $\psi$ will be used to obtain acoustic pressure $p$ and velocity $v$ via $p=-\rho_{0}(\partial / \partial t) \psi$ and $\vec{v}=\operatorname{grad} \psi$, where $\rho_{0}$ is the density of the undisturbed medium.

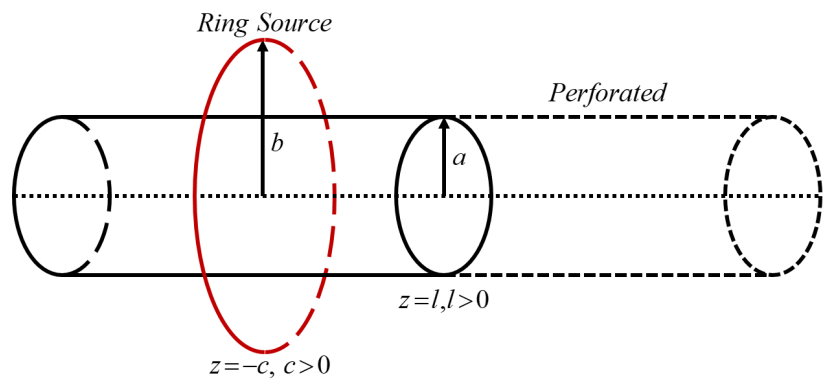

FiguRE 1. Geometry of the current problem. 
For analytical convenience, it is suitable to write the total field as follows

$$
\psi^{T}(r, z)= \begin{cases}\psi_{1}(r, z) ; & r>b \\ \psi_{2}(r, z) ; & a<r<b \\ \psi_{3}(r, z) ; & r<a\end{cases}
$$

Time dependence is assumed to be $e^{-i \omega t}$ and suppressed throughout this work where $\omega=2 \pi f$ is the angular frequency and $f$ is the frequency.

2.2. Derivation of the Wiener-Hopf System. The unknown fields $\psi_{1}(r, z), \psi_{2}(r, z)$ and $\psi_{3}(r, z)$ satisfy the wave equation for $z \in(-\infty, \infty)$

$$
\left[\frac{1}{r} \frac{\partial}{\partial r}\left(r \frac{\partial}{\partial r}\right)+\frac{\partial^{2}}{\partial z^{2}}+k^{2}\right] \psi_{j}(r, z)=0, j=1,2,3
$$

with wave number $k=\omega / c_{0}$ where $c_{0}$ is the speed of the sound. By taking Fourier transform of these three equations we obtain the following integral representations

$$
\begin{gathered}
\psi_{1}(r, z)=\frac{k}{2 \pi} \int_{L} A(\alpha) H_{0}^{(1)}(\lambda k r) e^{-i \alpha k z} d \alpha \\
\psi_{2}(r, z)=\frac{k}{2 \pi} \int_{L}\left[B(\alpha) J_{0}(\lambda k r)+C(\alpha) Y_{0}(\lambda k r)\right] e^{-i \alpha k z} d \alpha \\
\psi_{3}(r, z)=\frac{k}{2 \pi} \int_{L} D(\alpha) J_{0}(\lambda k r) e^{-i \alpha k z} d \alpha
\end{gathered}
$$

where $L$ is a suitable inverse Fourier transform integration contour in the complex $\alpha$-plane [13. $J_{0}$ and $Y_{0}$ are the Bessel and Neumann functions of order zero, respectively. $H_{0}^{(1)}=J_{0}+i Y_{0}$ is the Hankel function of the first type. $\lambda=\sqrt{1-\alpha^{2}}$ is square root function. The unknown coefficients $A(\alpha), B(\alpha), C(\alpha)$ and $D(\alpha)$, which are introduced in the solution of potential function, are to be determined by applying the following boundary conditions and continuity relations at $r=a$ and $r=b$.

$$
\begin{gathered}
\frac{\partial}{\partial r} \psi_{2}(a, z)=0, z<l \\
\frac{\partial}{\partial r} \psi_{3}(a, z)=0, z<l \\
\frac{\partial}{\partial r} \psi_{2}(a, z)=\frac{\partial}{\partial r} \psi_{3}(a, z), z>l \\
\psi_{2}(a, z)=\psi_{3}(a, z)+i \frac{\zeta_{p}}{k} \frac{\partial}{\partial r} \psi_{3}(a, z), z>l
\end{gathered}
$$

$\zeta_{p}$ which is given by [14], is the nondimensional specific acoustic impedance, describing the acoustic properties of the perforated cylinder.

$$
\zeta_{p}=\left[0.006-i k\left(t_{w}+0.75 d_{h}\right)\right] / \sigma
$$


where $\sigma$ is the porosity, $d_{h}$ is the perforate hole diameter and $t_{w}$ is the screen thickness. By using the ring source definition, given as

$$
\begin{gathered}
\frac{\partial}{\partial r} \psi_{1}(b, z)-\frac{\partial}{\partial r} \psi_{2}(b, z)=\delta(z+c),-\infty<z<\infty \\
\psi_{1}(b, z)=\psi_{2}(b, z), \quad-\infty<z<\infty
\end{gathered}
$$

where $\delta$ is dirac delta function. Applying the boundary conditions on $r=a$ for equations (6), (7) and (8) and taking Fourier transforms gives

$$
[D(\alpha)-B(\alpha)] J_{1}(\lambda k a)=C(\alpha) Y_{1}(\lambda k a)
$$

similarly for equation (7)

$$
-D(\alpha) \lambda k J_{1}(\lambda k a)=e^{i \alpha k l} \Phi^{+}(\alpha)
$$

continuity of pressure at $r=a$ for (9) yields

$$
[B(\alpha)-D(\alpha)] J_{0}(\lambda k a)+C(\alpha) Y_{0}(\lambda k a)=e^{i \alpha k l} \Phi^{-}(\alpha)+i \frac{\zeta_{p}}{k} e^{i \alpha k l} \Phi^{+}(\alpha)
$$

where $\Phi^{+}(\alpha)$ and $\Phi^{-}(\alpha)$ are a function analytic at the upper $(\operatorname{Im} \alpha>0$ or $\operatorname{Im} \alpha=$ 0 and $\operatorname{Re} \alpha>0)$ and lower $(\operatorname{Im} \alpha<0$ or $\operatorname{Im} \alpha=0$ and $\operatorname{Re} \alpha<0)$ half plane respectively and defined as

$$
\begin{gathered}
\Phi^{+}(\alpha)=\int_{l}^{\infty} \frac{\partial}{\partial r} \psi_{3}(a, z) e^{i \alpha k(z-l)} d z \\
\Phi^{-}(\alpha)=\int_{-\infty}^{l}\left[\psi_{2}(a, z)-\psi_{3}(a, z)\right] e^{i \alpha k(z-l)} d z
\end{gathered}
$$

The spectral coefficient $D(\alpha)$ can be found easily from (14) while $A(\alpha), B(\alpha)$ and $C(\alpha)$ are related to each other by the definition of the ring source given in $(11,12)$. By using the boundary conditions on $r=b$, one obtains

$$
\begin{gathered}
\lambda k A(\alpha) H_{1}^{(1)}(\lambda k b)=\lambda k B(\alpha) J_{1}(\lambda k b)+\lambda k C(\alpha) Y_{1}(\lambda k b)-e^{-i \alpha k c} \\
A(\alpha) H_{0}^{(1)}(\lambda k b)=B(\alpha) J_{0}(\lambda k b)+C(\alpha) Y_{0}(\lambda k b)
\end{gathered}
$$

where $H_{1}^{(1)}=J_{1}+i Y_{1}$. From (18) and (19), we get

$$
\begin{gathered}
B(\alpha)=A(\alpha)+e^{-i \alpha k c} \frac{\pi b}{2} Y_{0}(\lambda k b) \\
C(\alpha)=i A(\alpha)-e^{-i \alpha k c} \frac{\pi b}{2} J_{0}(\lambda k b)
\end{gathered}
$$


(13) and (14) allows us to express the coefficients $A(\alpha)$ and $D(\alpha)$ in terms of the analytic function $\Phi^{+}(u)$

$$
\begin{gathered}
A(\alpha)=-\frac{e^{i \alpha k l} \Phi^{+}(\alpha)}{\lambda k H_{1}^{(1)}(\lambda k a)}+\frac{\pi b}{2} \frac{e^{-i \alpha k c}}{H_{1}^{(1)}(\lambda k a)}\left[J_{0}(\lambda k b) Y_{1}(\lambda k a)-Y_{0}(\lambda k b) J_{1}(\lambda k a)\right] \\
D(\alpha)=-\frac{e^{i \alpha k l} \Phi^{+}(\alpha)}{\lambda k J_{1}(\lambda k a)}
\end{gathered}
$$

The substitution of $B(\alpha), C(\alpha)$ and $D(\alpha)$ given by (20), (21), (23) into (15) yields

$$
\Phi^{+}(\alpha) M(\alpha)=\Phi^{-}(\alpha)+\frac{b}{a} e^{-i \alpha k(c+l)} \frac{H_{0}^{(1)}(\lambda k b)}{\lambda k H_{1}^{(1)}(\lambda k a)}
$$

where $M(\alpha)$ is kernel function to be factorized

$$
M(\alpha)=\frac{J_{0}(\lambda k a)}{\lambda k J_{1}(\lambda k a)}-\frac{H_{0}^{(1)}(\lambda k a)}{\lambda k H_{1}^{(1)}(\lambda k a)}-i \frac{\zeta_{p}}{k}
$$

2.3. Solution of the Wiener-Hopf Equation. Consider the Wiener-Hopf equation in (24) and rearrange it using (25) in the following form

$$
\Phi^{+}(\alpha) M^{+}(\alpha)=\Phi^{-}(\alpha) M^{-}(\alpha)+\frac{b}{a} e^{-i \alpha k(c+l)} M^{-}(\alpha) \frac{H_{0}^{(1)}(\lambda k b)}{\lambda k H_{1}^{(1)}(\lambda k a)}
$$

Here, $M^{-}(\alpha)$ and $M^{+}(\alpha)$ are analytic functions in the lower and upper half planes, respectively, From the Wiener-Hopf factorization of $M(\alpha)$ as [15], one gets

$$
M(\alpha)=\frac{M^{+}(\alpha)}{M^{-}(\alpha)}
$$

Now consider (26), by using the classical decomposition procedure for complex term, one gets

$$
\Phi^{+}(\alpha) M^{+}(\alpha)=\Phi^{-}(\alpha) M^{-}(\alpha)+I^{+}(\alpha)+I^{-}(\alpha)
$$

Decomposing $I(\alpha)$ we obtain split functions $I^{+}(\alpha)$ and $I^{-}(\alpha)$ which are regular in the upper and lower half planes, respectively [16].

$$
I(\alpha)=\frac{b}{a} e^{-i \alpha k(c+l)} M^{-}(\alpha) \frac{H_{0}^{(1)}(\lambda k b)}{\lambda k H_{1}^{(1)}(\lambda k a)}=I^{+}(\alpha)+I^{-}(\alpha)
$$

The Wiener-Hopf equation in (28), yields

$$
\Phi^{+}(\alpha) M^{+}(\alpha)-I^{+}(\alpha)=\Phi^{-}(\alpha) M^{-}(\alpha)+I^{-}(\alpha)
$$

Now both sides of (30) are analytical functions on upper and lower regions, one can obtains the Wiener-Hopf solution

$$
\Phi^{+}(\alpha)=I^{+}(\alpha) / M^{+}(\alpha)
$$


and

$$
\Phi^{-}(\alpha)=-I^{-}(\alpha) / M^{-}(\alpha)
$$

\section{FAR Field}

The total field in $r>b$ can be evaluated from (3)

$$
\psi_{1}(r, z)=\frac{k}{2 \pi} \int_{L} A(\alpha) H_{0}^{(1)}(\lambda k r) e^{-i \alpha k z} d \alpha
$$

We may write the total field as follows

$$
\psi_{1}(r, z)=\psi_{d}(r, z)+\psi_{i}(r, z)+\psi_{r}(r, z)
$$

where

$$
\begin{gathered}
\psi_{d}(r, z)=-\frac{k}{2 \pi} \int_{L} \frac{\Phi^{+}(\alpha)}{\lambda k H_{1}^{(1)}(\lambda k a)} H_{0}^{(1)}(\lambda k r) e^{-i \alpha k(z-l)} d \alpha \\
\psi_{i}(r, z)+\psi_{r}(r, z)=\frac{k b}{4} \int_{L} \frac{J_{0}(\lambda k b) Y_{1}(\lambda k a)-Y_{0}(\lambda k b) J_{1}(\lambda k a)}{H_{1}^{(1)}(\lambda k a)} \\
\times H_{0}^{(1)}(\lambda k r) e^{-i \alpha k(z+c)} d \alpha
\end{gathered}
$$

Replacing $H_{0}^{(1)}(\lambda k r)$ by its asymptotic expressions valid for $k r \gg 1$.

$$
H_{0}^{(1)}(\lambda k r) \sim \sqrt{\frac{2}{\pi \lambda k r}} e^{i(\lambda k r-\pi / 4)}
$$

and applying the saddle point technique [17], we get

$$
\psi_{1}(r, z)=\psi_{d}\left(R_{1}, \theta_{1}\right)+\psi_{i}\left(R_{2}, \theta_{2}\right)+\psi_{r}\left(R_{2}, \theta_{2}\right)
$$

where

$$
\begin{gathered}
\psi_{d}\left(R_{1}, \theta_{1}\right)=\frac{i}{\pi} \frac{\Phi^{+}\left(-\cos \theta_{1}\right)}{\sin \theta_{1} H_{1}^{(1)}\left(k a \sin \theta_{1}\right)} \frac{e^{i k R_{1}}}{k R_{1}} \\
\psi_{i}\left(R_{2}, \theta_{2}\right)+\psi_{r}\left(R_{2}, \theta_{2}\right) \\
=\frac{k b}{2 i} \frac{J_{0}\left(k b \sin \theta_{2}\right) Y_{1}\left(k a \sin \theta_{2}\right)-Y_{0}\left(k b \sin \theta_{2}\right) J_{1}\left(k a \sin \theta_{2}\right)}{H_{1}^{(1)}\left(k a \sin \theta_{1}\right)} \frac{e^{i k R_{2}}}{k R_{2}}
\end{gathered}
$$

Here $R_{1}, \theta_{1}$ and $R_{2}, \theta_{2}$ are the spherical coordinates

$$
r=R_{1} \sin \theta_{1}, z-l=R_{1} \cos \theta_{1}
$$

and

$$
r=R_{2} \sin \theta_{2}, z+c=R_{2} \cos \theta_{2}
$$




\section{Numerical Results}

In order to show the effects of the parameters like the radii of the duct and the ring source and the perforated part on the diffracted field, some results displaying a changing of the sound pressure level with different values are presented in this section. Figures are plotted for Sound Pressure Level (SPL), described by

$$
S P L=20 \log _{10}\left|\frac{p}{2 \cdot 10^{-5}}\right|
$$

Far field values are plotted $46 \mathrm{~m}$ from the duct edge. Parameter values of the perforated part are taken from the study of [14].

First, variations of sound pressure level for different values of radius $(a)$ are presented in Figure 2 for $f=1500 \mathrm{~Hz}, b=0.075 \mathrm{~m}, l=0.010 \mathrm{~m}, c=0.050 \mathrm{~m}$, $t_{w}=0.00081 \mathrm{~m}, d_{h}=0.0249 \mathrm{~m}, \sigma=0.057$. In the graph, it is seen that as the value of $(a)$ decreases, the sound pressure level decreases. In Figure 3, similar analysis is

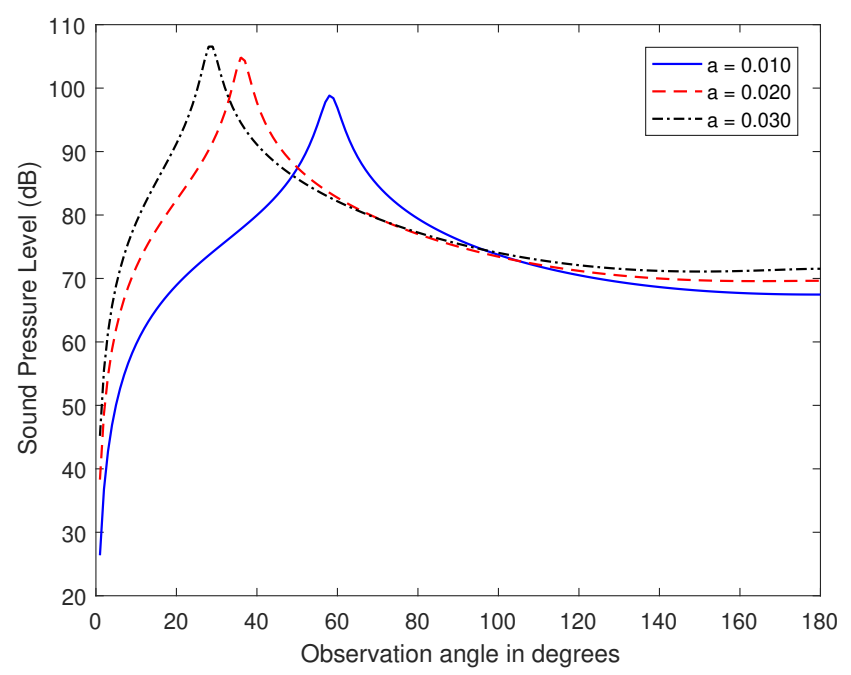

FiguRE 2. SPL versus the observation angle for different values of the duct radius $(a)$ with $f=1500 \mathrm{~Hz}, b=0.075 \mathrm{~m}, l=0.010$ $\mathrm{m}, c=0.050 \mathrm{~m}, t_{w}=0.00081 \mathrm{~m}, d_{h}=0.0249 \mathrm{~m}, \sigma=0.057$.

also carried for different values of ring source radius $(b)$ for $f=1500 \mathrm{~Hz}, a=0.010$ $\mathrm{m}, l=0.010 \mathrm{~m}, c=0.050 \mathrm{~m}, t_{w}=0.00081 \mathrm{~m}, d_{h}=0.0249 \mathrm{~m}, \sigma=0.057$. Sound pressure level decreases with decreasing value of ring source radius like in Figure 2.

Figures 4 and 5 display the same effect to the sound pressure level for different values of $l$ and $c$. For Figure 4, the parameter values are $f=1500 \mathrm{~Hz}, a=0.010$ 


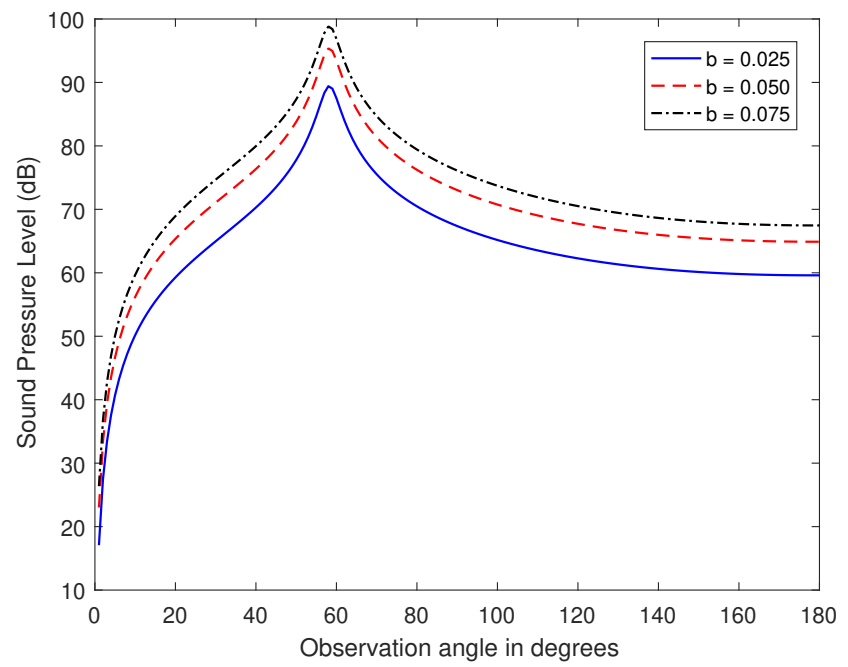

FigURE 3. SPL versus the observation angle for different values of the ring source radius ( $b$ ) with $f=1500 \mathrm{~Hz}, a=0.010 \mathrm{~m}, l=0.010$ $\mathrm{m}, c=0.050 \mathrm{~m}, t_{w}=0.00081 \mathrm{~m}, d_{h}=0.0249 \mathrm{~m}, \sigma=0.057$.

$\mathrm{m}, b=0.0510 \mathrm{~m}, c=0.050 \mathrm{~m}, t_{w}=0.00081 \mathrm{~m}, d_{h}=0.0249 \mathrm{~m}, \sigma=0.057$ while for Figure 5 the parameter values are $f=1500 \mathrm{~Hz}, a=0.010 \mathrm{~m}, b=0.050 \mathrm{~m}$, $l=0.010 \mathrm{~m}, t_{w}=0.00081 \mathrm{~m}, d_{h}=0.0249 \mathrm{~m}, \sigma=0.057$. Sound pressure level decreases with increasing values of $l$ and $c$, as expected.

From Figure 6, one can see the effect of the frequency on the sound pressure level. This graph is plotted for $a=0.010 \mathrm{~m}, b=0.025 \mathrm{~m}, l=0.010 \mathrm{~m}, c=0.050$ $\mathrm{m}, t_{w}=0.00081 \mathrm{~m}, d_{h}=0.0249 \mathrm{~m}, \sigma=0.057$. The similar effect is observed like in Figure 2.

In Figure 7, the effect of specific acoustic impedance $\left(\zeta_{p}\right)$ on sound pressure level is first studied for three different acoustic impedances and compared to open case. This graph is plotted for non dimensional parameters which values are $k a=1$, $k b=10, k l=10, k c=6$. It is seen that existing of perforated part makes contribution to the reduction of sound pressure level and when imaginary coefficient of acoustic impedance decreases the pressure level decreases. It should be noted that, due to Equation (10), the real part of $\zeta_{p}$ remains constant and the imaginary part changes for different values of frequency.

In order to show the accuracy of the numerical results obtained in this study, $\zeta_{p}$ is taken zero and the results are compared with the study of [12] for semi-infinite rigid duct. Figure 8 shows that the results are consistent, that is, the results obtained 


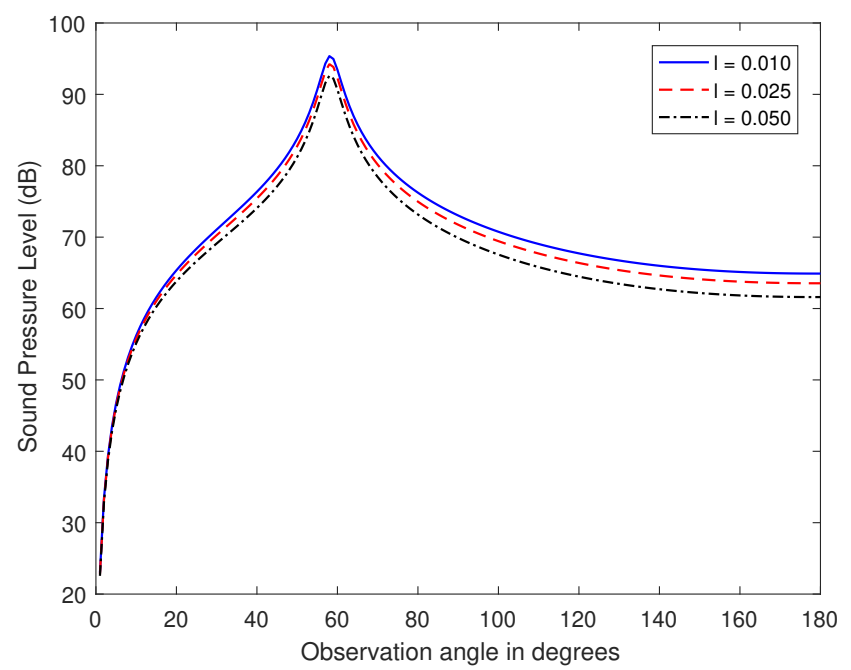

FiguRE 4. SPL versus the observation angle for different values of the duct extension $(l)$ with $f=1500 \mathrm{~Hz}, a=0.010 \mathrm{~m}, b=0.0510$ $\mathrm{m}, c=0.050 \mathrm{~m}, t_{w}=0.00081 \mathrm{~m}, d_{h}=0.0249 \mathrm{~m}, \sigma=0.057$.

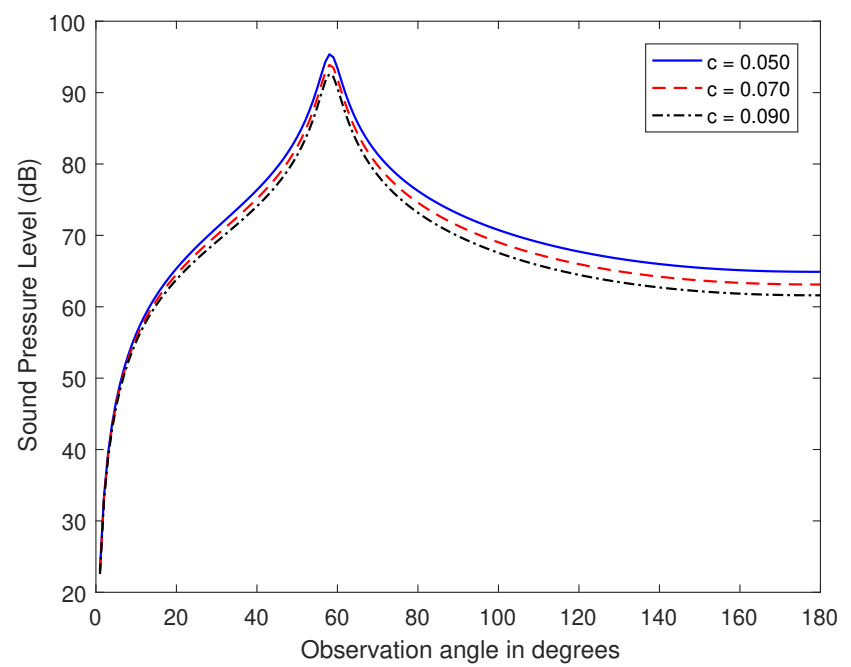

FiguRE 5. SPL versus the observation angle for different values of the ring source axes $(c)$ with $f=1500 \mathrm{~Hz}, a=0.010 \mathrm{~m}, b=0.050$ $\mathrm{m}, l=0.010 \mathrm{~m}, t_{w}=0.00081 \mathrm{~m}, d_{h}=0.0249 \mathrm{~m}, \sigma=0.057$. 


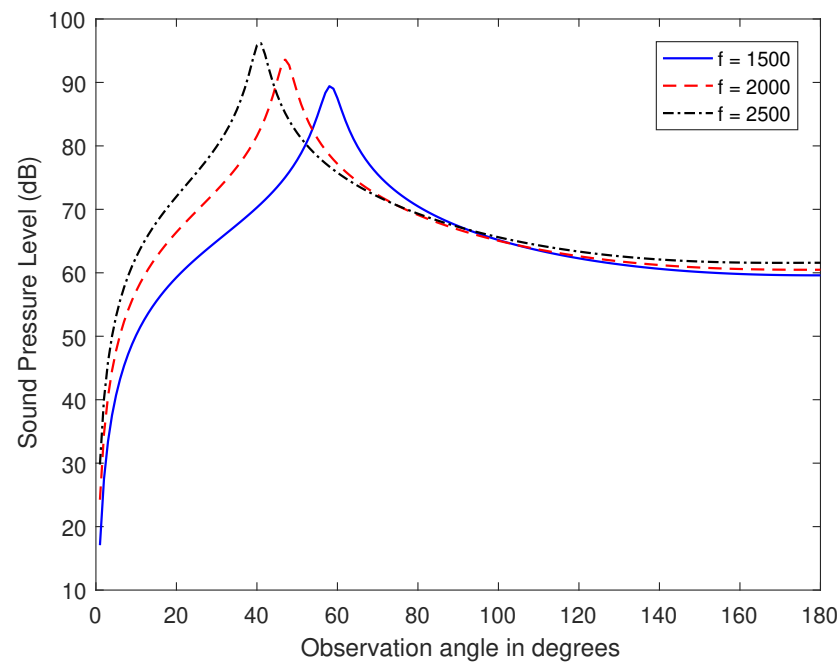

Figure 6. SPL versus the observation angle for different values of the frequency $(f)$ with $a=0.010 \mathrm{~m}, b=0.025 \mathrm{~m}, l=0.010 \mathrm{~m}$, $c=0.050 \mathrm{~m}, t_{w}=0.00081 \mathrm{~m}, d_{h}=0.0249 \mathrm{~m}, \sigma=0.057$.

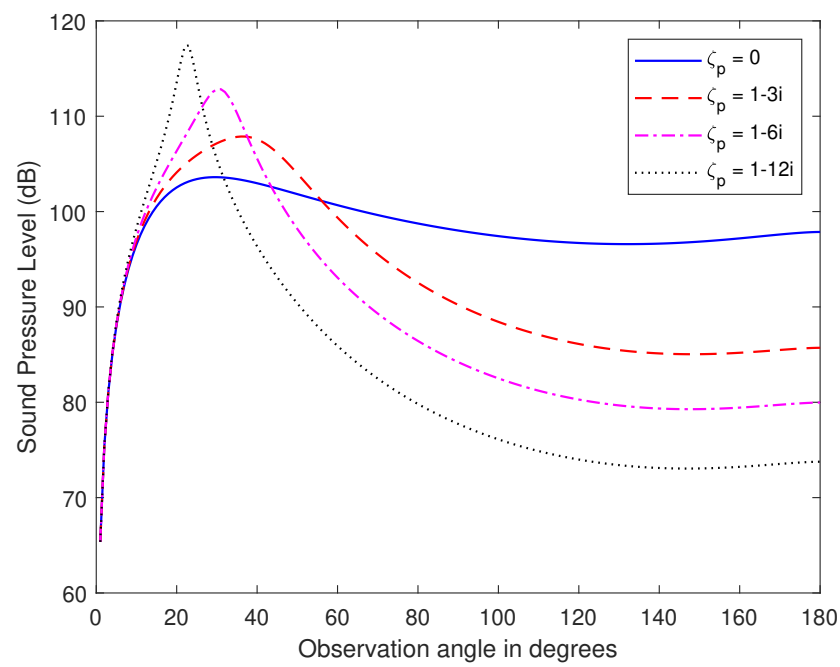

FiguRE 7. SPL versus the observation angle for open perforated duct with $k a=1, k b=10, k l=10, k c=6$. 


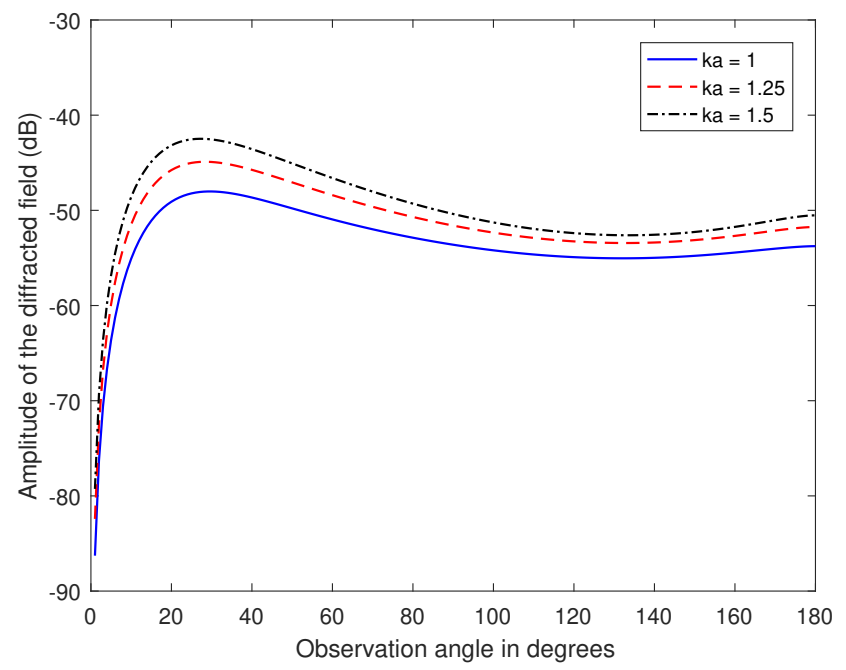

Figure 8. Comparison of the diffracted field with the study of 12 for semi infinite rigid duct.

in this study are correct. Notice that for Figure 8, the diffracted field graphic is produced for $20 \log _{10}\left|\psi_{d}\left(R_{1}, \theta_{1}\right)\right|$.

\section{Conclusion}

In this study, diffraction of sound waves emanating from a ring source by an infinite cylindrical duct which is rigid for $z<l$ and perforated for $z>l$ has been investigated by using the Fourier transform technique in conjunction with the Wiener Hopf technique. The problem is modelled two dimensional due to symmetry of the geometry and of the ring source. A solution is derived by solving the Wiener Hopf equation. To a better understanding the effect of the parameters of the problem such as the radii of the duct and ring source and perforated part on the sound pressure level, graphics are presented for some specific values of the problem. It has been observed that the sound pressure level decreases as the values of the frequency $(f)$, the duct radius $(a)$ and the ring source radius $(b)$ decreases. On the contrary, it is observed that the sound pressure level increases as the values of the duct extension $(l)$ and the ring source axes $(c)$ decreases. The effect of the perforated duct on the sound pressure level is more effective. While a few decibels change in sound pressure level is observed for other parameters of the problem, the variation from the perforated duct is more significant. It is found that presence of the perforated part reduced the sound pressure level when compared with the open part situation. The results are also compared with the study of 12$]\left(\zeta_{p}=0\right)$ and 
it is observed that the agreement is perfect.

Declaration of Competing Interests The author has no competing interests to declare.

\section{REFERENCES}

[1] Levine, H., Schwinger J., On the radiation of sound from an unflanged circular pipe, Physical Review, 73 (1948), 383-406. https://doi.org/10.1103/PhysRev.73.383

[2] Noble, B., Methods Based on the Wiener-Hopf Techniques, Pergamon Press, London, 1958.

[3] Rawlins, A.D., Radiation of sound from an unflanged rigid cylindrical duct with an acoustically absorbing internal surface, Proc. Roy. Soc. Lond. A. 361 (1978), 65-91. https://doi.org/10.1098/rspa.1978.0092

[4] Buyukaksoy, A., Polat, B., Diffraction of acoustic waves by a semi-infinite cylindrical impedance pipe of certain wall thickness, Journal of Engineering Mathematics, 33 (1998), 333-352. https://doi.org/10.1023/A:1004301829276

[5] Tiryakioglu, B., Diffraction of sound waves by a lined cylindrical cavity, International Journal of Aeroacoustics, 19(1-2) (2020), 38-56. https://doi.org/10.1177/1475472X20905043

[6] Nilsson, B., Brander, O., The propagation of sound in cylindrical ducts with mean flow and Bulk-reacting lining I. modes in an infinite duct, J. Inst. Maths. Applics, 26 (1980), 269-298. https://doi.org/10.1093/imamat/26.3.269

[7] Yang, C., Cheng, L., Hu, Z., Reducing interior noise in a cylinder using micro-perforated panels, Applied Acoustics, 95 (2015), 50-56. https://doi.org/10.1016/j.apacoust.2015.02.003

[8] Wang, J., Rubini, P., Qin, Q., Application of a porous media model for the acoustic damping of perforated plate absorbers, Applied Acoustics, 127 (2017), 324-335. https://doi.org/10.1016/j.apacoust.2017.07.003

[9] Tiryakioglu, B., Radiation of acoustic waves by a partially lined pipe with an interior perforated screen, Journal of Engineering Mathematics, 122(1) (2020), 17-29. https://doi.org/10.1007/s10665-020-10042-x

[10] Tiryakioglu, B., Radiation of sound by a coaxial waveguide with semi-infinite perforated duct, Waves in Random and Complex Media. https://doi.org/10.1080/17455030.2020.1782511

[11] Tiryakioglu, B., Analysis of Sound Waves with Semi Perforated Pipe, 7th International Symposium on Innovative Technologies in Engineering and Science, Sanliurfa, Turkey, (2019), 704-710.

[12] Tiryakioglu, B., A Note on Ring Source over Semi-Infinite Rigid Pipe, International Journal of Advances in Engineering and Pure Sciences, 31(2) (2019), 133-139. https://doi.org/10.7240/jeps.488305

[13] Rienstra, S. W., Peake. N., Modal Scattering at an Impedance Transition in a Lined Flow Duct, 11th AIAA/CEAS Aeroacoustics Conference, Monterey, CA, USA, (2005) 1-19.

[14] Sullivan, J. W., Crocker, M. J., Analysis of concentric-tube resonators having unpartitioned cavities, Journal of the Acoustical Society of America, 64 (1978), 207-215. https://doi.org/10.1121/1.381963

[15] Demir, A., Rienstra, S. W., Sound Radiation from a Lined Exhaust Duct with Lined Afterbody, 16th AIAA/CEAS Aeroacoustics Conference, Stockholm, Sweden, (2010) 1-18.

[16] Polat, B., Diffraction of Acoustic Waves by a Cylindrical Impedance Rod of Finite Length, Journal of Applied Mathematics and Mechanics, 79 (1999), 555-567. https://doi.org/10.1002/(SICI)1521-4001(199908)79:8¡555::AID-ZAMM555¿3.0.CO;2-

[17] Mittra, R., Lee, S. W., Analytical techniques in the theory of guided waves, The Macmillan Company, 1971. 